\title{
Automatic fuel tank monitoring, tracking \& theft detection system
}

\author{
Komal D/o Shoukat Ali Khuwaja ${ }^{1, *}$, Brohi Arif Ali², Vlad O. Mihalca ${ }^{1}$ and Radu Cătălin Țarcă \\ ${ }^{1}$ Mechatronics Department, University of Oradea, Romania \\ ${ }^{2}$ Electronics Department, MUET Jamshoro, Pakistan
}

\begin{abstract}
This paper presents the design, implementation and characterization of a hardware platform for Fuel Management System (FMS) for fuel carrying vehicles. The primary design goal is to devise a system capable of monitoring the fuel level in real time. This system is proposed for fuel carrying road tankers which carry fuel from oil depots to end users such as petrol-stations. This system is based on hardware as well as software. The hardware part consists of fuel level circuits, on-board Arduino, GSM and GPS modules. While the software part consists of LabView for Database purposes. This system offers an Automatic Electronic System (AES) which replaces manual monitoring of fuel. This system measures fuel volume and sends measured volume to the owner's mobile as well as owner's computer through GSM network. It also provides a technique for detecting theft or fraud incidents in case of fuel theft or fuel leakage. Additionally the system can track the location of each fuel carrying tanker in order to locate the place of any incident that could have occurred. This system allows for an automated analysis and monitoring of fuel level, having a reduced cost due to affordable and easy-to-acquire electronic components.
\end{abstract}

\section{Introduction}

Fuel Management Systems (FMS) are used to maintain, control and monitor the fuel consumption in any type of organization that uses transport, including: rail, road, air and water as means of business. Currently the organizations are facing a serious problem of managing the fuel transportation due to manual monitoring. This manual monitoring provides an inefficient way of calculating and analysing the fuel ingestion and can lead to financial losses for the company.

In the typical scenario used by most organizations there is no logging or auditing mechanism to check that the number of litres written on the receipt correspond to the actual amount of fuel in the tank. The driver takes the vehicle to the fuel depot, fills the tank and gets the receipt from the pump manager, mentioning the number of litres of fuel inserted into the vehicle tank, date and the amount. The driver gives this receipt to the person in charge of the organization, which keeps up the record and sends the amount to the pump-owner at the end of the month.

This scenario is affected by many possible flaws: the driver does not know whether the amount of fuel inserted in the fuel is the same as the amount declared on the receipt. Another flaw of the system is caused by misleading information created by the pump station manager. A third example would be the involvement of the staff from company's management personnel which commit fraud by falsifying information regarding the amount of fuel inserted.

Many companies must keep all the above mentioned factors into account, therefore the need for an automated electronics system (AES) that not only checks the fuel ingested but also digs out the theft of fuel in order to save company from loss [10]. Furthermore, this system provides an efficient way of tracking vehicles in order to localize the fuel Tankers as well as a software database for fuel records, residing on end user's workstations or mobile phones [11]. The database contains the number of fuel litres at the time of insertion and number of fuel thefts when the volume of tanker changes a lot before reaching the destination.

Fuel transportation or fuel delivery to the sites takes place through various ways such as approved highway tanks, heavy ships, trains and through pipelines. This system only focuses on highway fuel tankers for the transportation of fuel on the subject of management. The System offers the most comprehensive solution on the market for knowing exactly how much fuel is inserted and how much is lost. From complete fuel accountability to total reconciliation and protection against fuel theft, this keeps our assets covered.

This system provide an efficient, cost effective and automated fuel management system that is especially customized for the companies of Pakistan that view stealing fuel as a serious problem but due to costly existing solutions, it is difficult for them to afford the systems already available on the market.

\subsection{Fuel Management System (FMS)}

GPS Fleet Tracking is an all-in-one management solution that can be used to monitor, manage, and recover assets. By tracking valuables, we can better manage and monitor

"Corresponding author: komalali_89@yahoo.com 
their whereabouts, cutting down on wasted time and fuel consumption. With GPS Fleet Tracking information, we can evaluate performance and cut down on extra costs. This enhances the company's value by improving the level of service provided, leading to higher profits and customer satisfaction.

This automatic Fleet Tracking reports are easy-to-read graphs that let us identify operational trouble spots and improve fleet management [1].

\subsection{Automatic Fuel Management System (AFMS)}

Automatic Fuel Management System (AFMS) consists of basic electronic components which are then mounted on highway tankers for the purpose of measurement and monitoring of its volume. It measures the number of fuel litres when inserted into the tank as well as when fuel is stolen or leaked and then it sends that measured data for analysis and additional statistics.

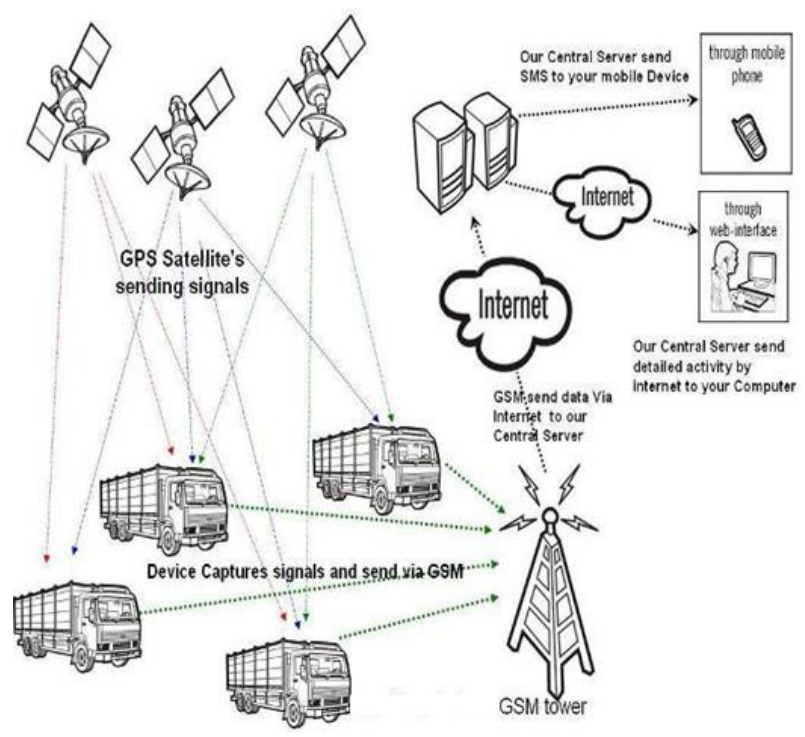

Fig. 1. Automatic Fleet Management System (AFMS)

According to Wikipedia a "GPS Fleet Tracking Is An All-In-One Management Solution That Lets You Monitor, Manage, And Recover Assets". By tracking your valuables, you can better manage and monitor their whereabouts, cutting down on wasted time or unnecessary fuel [1].

M. Saraswati in "Design and construction of water level measurement system" states that water measurement data is very important in some water related fields. An automatic water measurement system is needed which prevent the difficulties when one does measurement manually [2].

M. Saravanan, in his paper "Tracking Anomalies in Vehicle Movements using Mobile GIS" stated that detecting fraud activities and anomalies in the movement of vehicles are the two major concerns for the vehicle fleet industry. Some of these which incur heavy losses to the organization include fuel theft, loading and unloading related frauds, route variations, inefficient fuel maintenance and frequent breakdowns [3][4].

\section{Methodology}

The System may consist of hardware part as well as software part. Hardware part is used for sensing number of litres and used for communication of data processing whereas software part is used for the representation of data. The suggested system mainly consists of 3 modules namely embedded system module, GSM communication module and GPS tracking module. This structure is similar to the one presented in [5] and [7]. The first module contains fuel level sensor with a microcontroller which is placed in each fuel tankers to detect the number of litres and to interact with the GSM communication network.

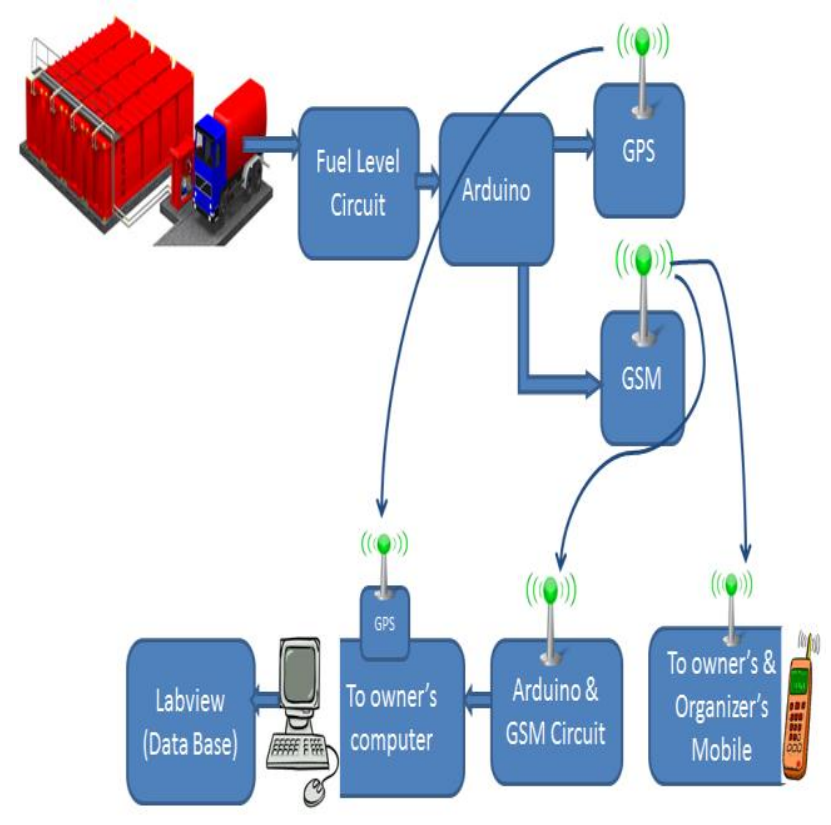

Fig. 2. General Block Diagram

The embedded system module acquires information from the fuel vehicles, maintains record and sends messages to the monitoring agents through communication module. The GPS tracking module is used for the real time tracking of fuel tankers [6] .

\subsection{Embedded System Module}

Embedded system module is composed of fuel level sensor and a processing unit known as Arduino Mega 2560. In order to measure tanker volume the distance sensor HC-SR04 is used which works by sending out a pulse of ultrasonic sound and measuring the amount of time it takes for the sound to come back after hitting the fuel surface [2]. The sensor is implemented with Arduino controller for the purpose of measuring and processing the distance above fuel level. 


\subsection{Communication Module}

This system mainly depends on the communication module for sending and monitoring of data wirelessly. The measured data is sent remotely for further analysis [8]. A similar UDP-based communication scheme can be seen in [9]. Our system makes use of AT commands sent through the Arduino GSM module.

\subsection{The prototype Container}

The container is made up of plastic which has dimensions 18 inch length, 12 inch width and 12 inch height. It has holes at upper portion of container for placing ultra-sonic sensors for measuring fuel level within the container. The container is shown in figure below.

Fig. 3. Container measurement

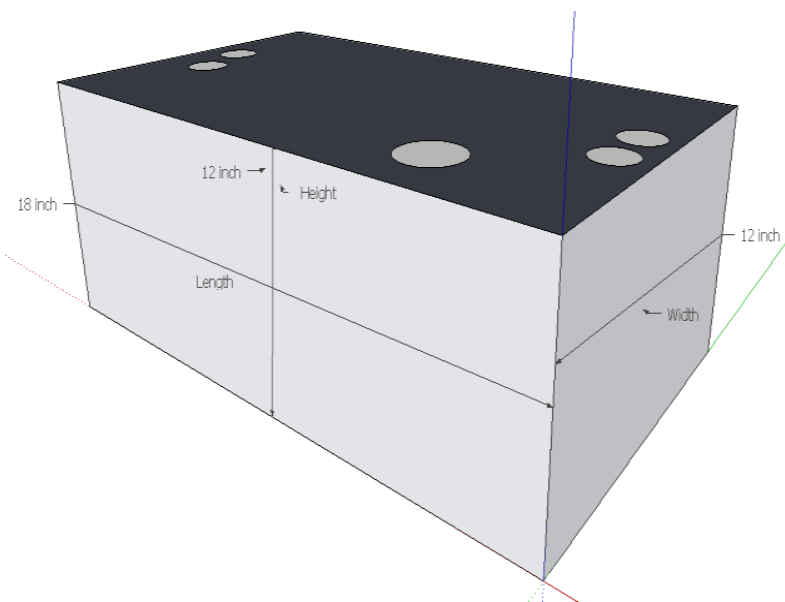

Total volume $=((1296.08) \times$ height $) / 1000)$ litters

Note: The above formula calculates the total volume of container whereas the height is measured by the Ultrasonic sensor and multiplied with the constant which is the overall dimensions of the container

\subsection{System software}

Software part is comprised of LABVIEW. It is a system design platform and development environment for a visual programming language from National Instruments. It comprises of database which is holding vehicle's number plate, total volume of the tanker, number of fuel litres inside the vehicle's tanker, number of lost fuel litres, total number of fuel litres carried in a month, total price of the fuel in a month and total loss/profit in a month.

LABVIEW integrates seamlessly with thousand of different hardware devices, and helps save development time with convenient features and a consistent programming framework across all hardware [2]. The flowchart depicts the procedure for receiving message through GSM modem into LABVIEW environment and hence extracting the data from it.

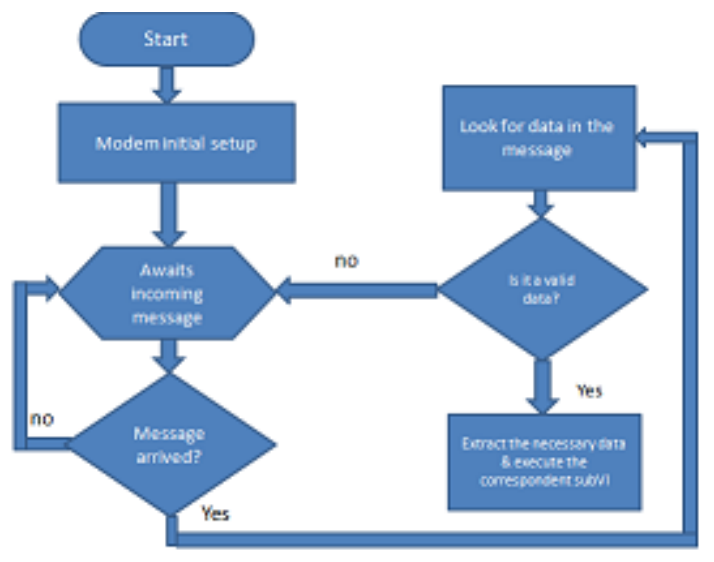

Fig. 4. Flow chart of message receiving

The database contains the data of all specified fuel tankers, each having individual system for the monitoring of fuel. The tanker truck and fuel within it is automatically displayed in LABVIEW just after receiving a message. The level indicator shows level of fuel inserted into the fuel tanker and map shows the location of the truck. This is shown below.

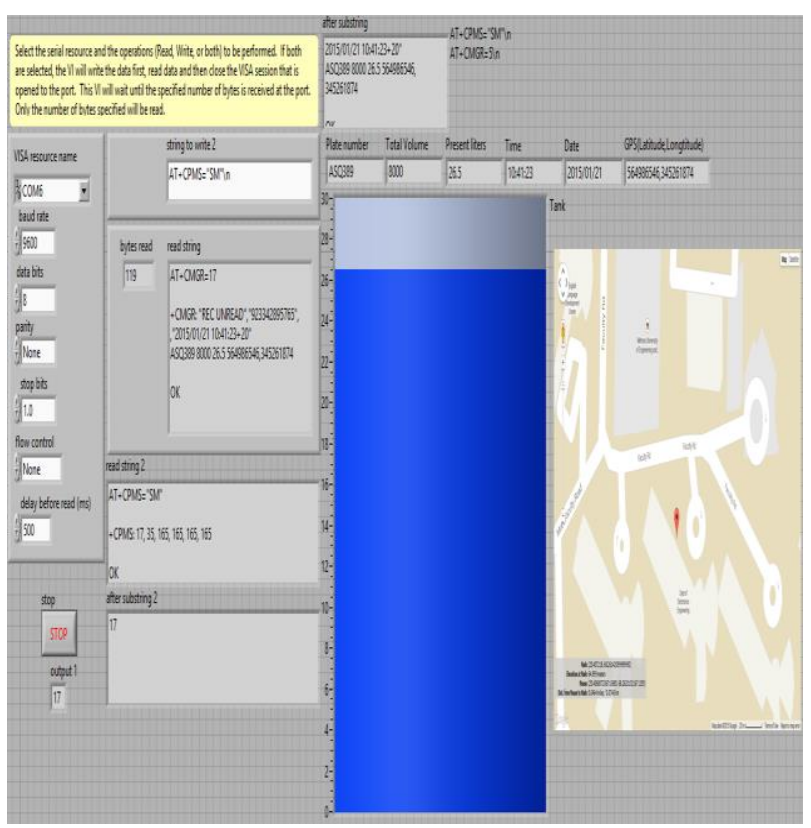

Fig. 5. Final view

\section{Survey}

We created a survey from different Petrol pumps and some other organizations and these are some questions and the results we found. In Fig. 6 first row is the answer to the first question and so on. According to the survey results, most of the people want this system in their practical life in order to obtain accurate information.

- Are you sure whether the number of fuel litres mentioned on the receipt is filled into the tanker or not?

- Have you ever caught the pump-employer doing fraud by filling the tanker with less fuel than required? 
- Do you want an automatic system for your organization which could keep record of fuel being transported in an automatic manner?

- Do you want an automated system which could calculate and send on your cell phone as well as personal computer the measured litres within the tank along with the amount specified on the receipt and the vehicle plate?

- Do you want an automated system which could track all your fuel tankers and inform you about incidents involving any of them on your computer?

- Do you want an automated system which could prevent the fuel theft/fraud or leakage and could calculate and analyse the number of wasted litres of fuel in a month incidents?

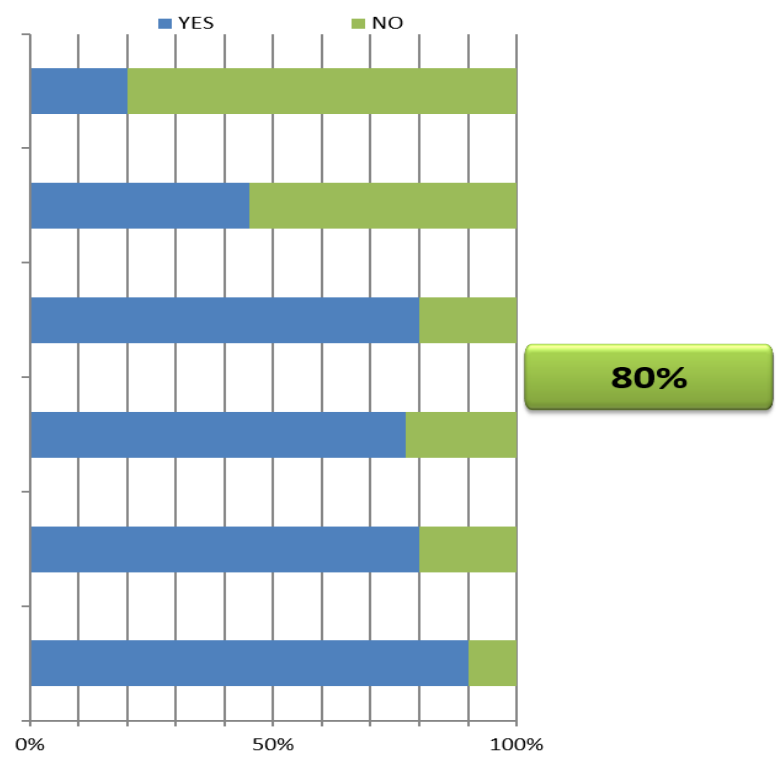

Fig. 6. Survey chart

\section{Conclusion}

This investigation presents and discusses the problems related to fuel management systems and overcoming these problems through an automated electronic system. The article presents considerations about both the hardware and software part of the system which involves measurement of fuel litres through fuel level circuit and sending these measurements through GSM to the Organizer's mobile as well as to the computer wirelessly.

The theoretical results show that $90 \%$ people are facing problems in managing and analyzing the fuel consumption through manual monitoring. Hence, there is a need of such a system which could minimize the problems occurring in fuel management and which could also analyze and calculate fuel consumption in an automated way. Although some systems are available regarding such applications, they are expensive hence organizations do not consider investing into them after comparing the fuel consumption and the price of such systems. The proposed system is designed by using easily available and cheap electronic components hence beneficial for any organization or industry having vehicles as key resources.
Transportation being an important resource for an Organization can have great impact on the Organization's economy. Management of the fuel involved by the logistics resource is of great importance. As a current practice it is done by Manual Monitoring which consists of Organizations having an agreement with nearby Petrol/Diesel suppliers on a monthly basis. Sometimes Manual Monitoring leads the Organization to financial losses by printing the wrong amount of fuel litres on the receipt. Such a scenario endorses an automated system which could allow for automated Monitoring and Analyzing of fuel.

\section{References}

1. Fleet Management Systems from http://gpsintegrated.com/services/fleet-managementsystem

2. M. Saraswati, E. Kuantama, P. Mardjoko, Eur. Symp. Comp. Model. Sim., Design and Construction of Water Level Measurement System Accessible through SMS, Vol. 6., 49-53, (2012)

3. M. Saravanan, S. Aishwarya, L. N. Aravindan, Sci \& Inf. Conf., Tracking anomalies in vehicle movements using mobile GIS, (2013)

4. T. Le-Tien, V. Phung, The IEEE International Symp. on Es. Design, Test \& Apps., Routing and Tracking System for Mobile Vehicles in Large Area, Vol.5 297300,(2010)

5. M. Popa, B. Suta, TELFOR, A solution for tracking a fleet of vehicles, Vol.19,1558 -1561, (2011)

6. G. Yuan, Z. Zhang, W. ShangGuan, International Conference on Internet Computing in Science and Engg., Research and Design of GIS in Vehicle Monitoring System, 223-228, (2008)

7. H. Hu, L. Fang, Inter. Sympo. on Inte. Infor. Techno. Apps., Design and Implementation of Vehicle Monitoring System Based on GPS/GSM/GIS, Vol.3, 278 - 281, (2009)

8. P .Chen, S. Liu, ICIE, Intelligent Vehicle Monitoring System Based on GPS, GSM and GIS, Vol.4, 38-40, (2010)

9. D. Liu, Z. Liang, Y. Li, H. Yang, FITME, Research and design of a high performance GPS vehicle monitoring system,Vol.3,59-62,(2010)

10. T. Enomoto, M. Sekine, S. Chikara, K. Kubota, Intelligent Transportation Systems, A freight status management system with restriction to record loading/unloading information by location, Vol.3, $548-553,(2003)$

11. A. Tahat, A. Said, F. Jaouni, W. Qadamani, ISCE, Android-based universal vehicle diagnostic and tracking system, Vol.16, 137-143, (2012) 